\title{
Positive functioning inventory: initial validation of a 12-item self-report measure of well-being
}

\author{
Stephen Joseph ${ }^{1 *}$ and John Maltby ${ }^{2}$
}

\author{
* Correspondence: \\ stephen.joseph@nottingham.ac.uk \\ ${ }^{1}$ School of Education, University of \\ Nottingham, NG8 1BB, UK \\ Full list of author information is \\ available at the end of the article
}

\begin{abstract}
Background: This paper describes the validation of the Positive Functioning Inventory (PFI-12). This is a 12-item self-report tool developed to assess a spectrum of functioning ranging from states of mental distress to states of well-being.

Method: Two samples (Sample 1: $N=242$, mean age $=20$ years. Sample 2: $N=301$, mean age $=20$ years) completed self-report measures of personality and social, physical and psychological functioning.

Results: Evidence is provided for internal-consistency reliability, test-retest reliability, incremental validity, and convergent and discriminant validity in relation to a number of other measures of personality, social, physical and psychological functioning.

Conclusion: The tool promises to be useful to practitioners and researchers who wish to assess positive psychological functioning.
\end{abstract}

Traditionally, the aim of clinical psychology has been to help alleviate distress and dysfunction, most commonly, depression and anxiety (Layard 2006). With the advent of positive psychology there is interest in how therapists can not only alleviate these negative states but also promote positive functioning defined in a way that goes beyond simply being the absence of these negative states (Joseph \& Linley 2006). The aim is to construct a new positive clinical psychology measure to assess a continuum of well-being.

The majority of general assessment tools in clinical practice are concerned with depressive and anxious states. For example, the Depression Anxiety and Stress Scales (DASS: Lovibond \& Lovibond 1995), Hospital Anxiety and Depression Scales (HADS: Zigmond \& Snaith 1983) and the Duke Anxiety-Depression (DUKE-AD: Parkinson 2002) as well as many measures specifically developed to assess either depressive or anxious states such as the Beck Depression and Anxiety Scales (Beck et al. 1988; Beck et al. 1961). Such tools aid assessment and treatment and can be used by therapists to monitor their client's progress and by researchers to evaluate clinical efficacy (see also, Fischer \& Corcoran 2007).

Since the emergence of positive psychology (Seligman \& Csikszentmihalyi 2000) it has however become accepted that the absence of psychological distress and dysfunction does not equate to the presence of positive functioning (e.g., Maddux et al. 2004). Low scores on measures that indicate the absence of depressive and anxious states do not indicate the presence of positive states of mind. For example, one of the most prominent measures is the Beck Depression Inventory (Beck et al. 1961). Scores on the BDI have a

(c) 2014 Joseph and Maltby; licensee Springer. This is an Open Access article distributed under the terms of the Creative Commons Attribution License (http://creativecommons.org/licenses/by/4.0), which permits unrestricted use, distribution, and reproduction in any medium, provided the original work is properly cited. 
potential range of 0 to 63. A score of 63 indicates intense depressive experience. A score of zero on the BDI indicates the absence of depressive experiences, as would be expected given its exclusive pathological wording, but a score of zero does not indicate the presence of positive functioning. For two people scoring zero, one could be high on positive functioning, the other low (Joseph \& Lewis 1998). By itself the BDI does not provide a measure of positive functioning.

Positive therapeutic approaches refer to clinical practices concerned with the facilitation of optimal functioning as well the alleviation of distress and dysfunction (Joseph \& Linley 2006). It may not be apparent at first why clinicians should be interested in positive functioning, but there are a number of reasons. First, the benefits of well-being include better physical health (Chida \& Steptoe 2008; Howell et al. 2007), improved social and occupational functioning (Pavot \& Diener 2004), and a decreased likelihood of future psychopathology (Wood \& Joseph 2010). Second, as with traditional measures of dysfunction, feedback on positive functioning is also likely to be helpful to both clients and therapists (Rashid \& Ostermann 2009). Third, by fostering engagement, meaning and positive emotions as a therapeutic aim it may also be helpful in treating current disorders such as depression (Seligman et al. 2008; Seligman et al. 2005).

There are a number of new dedicated general purpose positive psychology measures that assess positive functioning. For example, the Subjective Happiness Scale (Lyubomirsky \& Lepper 1999), and the Warwick-Edinburgh Mental Well-Being Scale (WEMWBS: Tennant et al. 2007) are two widely used measures. Others include the Positive and Negative Affect Scales (PANAS; Watson et al. 1988), the Scales of Psychological Well-being (Ryff \& Keyes 1995), and the Satisfaction With Life Scale (Diener et al. 1985). Despite the popularity of such tools among positive psychologists and others interested in human flourishing, their use by therapeutic practitioners and researchers is limited. One limitation of such measures is that the language they use is inconsistent with that of clinicians whose primary concern is the alleviation of negative states. For example, the WEMWBS consists only of positively worded items and is therefore unable to assess the degree of distress and dysfunction commonly required of clinical tools. Nonetheless, such measures can be useful for those working in a positive therapeutic way, but only when used alongside traditional measures. This, however, can add to the burden on the client and can detract from the clinical time available. As such there is a need for a new short general purpose tool that practitioners and researchers can use to assess not only the presence of positive functioning but also the traditional concerns of therapists.

As already noted, in clinical practice and research the two most widely assessed affective states are depression and anxiety. Psychiatry regards clinical depression and anxiety as discrete disorders separate from normal states. Psychologists, on the other hand, have tended to adopt a continuity model. In other words, clinical states of depression and anxiety and milder versions of these states may be seen as both falling on the same conceptual continuums. On this basis, much experimental work with mild depressive and anxious populations has informed our understanding and treatment of these clinical states. As such, an assumption we make in this paper is that depressive and anxious states can be understood using a continuity model.

The view that depressive and anxious states can be viewed as continuums raises the question of how we define the poles of these continuums. Mainstream psychology generally regards depressive and anxious states as separate dimensions to positive functioning. 
However, an alternative positive psychological view is that these dimensions reside on a continuum with states of positive functioning.

The view that depressive and anxious states represent one pole with states of positive functioning at the other pole is consistent with the circumplex model of emotions (Russell 1980; Russell \& Barrett 1998). The circumplex model of emotions is defined by the two dimensions of pleasantness-unpleasantness and high activation-low activation. Seen this way, depression is a state of low pleasantness and low activation and anxiety is a state of low pleasantness and high activation. The opposite state to depression is therefore characterised by high pleasantness and high activation. The opposite state to anxiety is characterised by high pleasantness and low activation. Although the circumplex of emotions has attracted much attention in emotion research (Ekkekakis 2013), its application to positive clinical psychology has been limited (see, Joseph \& Wood 2010). The implication, however, is that depression and anxiety may be represented by dimensional structures continuous with these polar opposite states.

Within positive psychology the notion that well-being can be represented on a continuum is widely accepted. There are two versions of the continuum approach, first, a "weak" version, which sees positive and negative functioning to be separate continuums, but both to be subsumed by a higher order well-being continuum; and, second, a "strong" version which sees affective states to exist on continuums. The weak version is widely accepted, although the strong version is a matter of continued debate (see Joseph \& Wood 2010). In the weak version, the concept of subjective well-being (SWB) provides a hierarchically organized conception (Diener et al. 1999). At the highest level of the hierarchy is a latent well-being variable which is a continuum ranging from extreme low to extreme high well-being. At the next level of the hierarchy are positive affect, negative affect, and high satisfaction with life. Each of these three variables can be broken down further at the next level of the hierarchy, with positive affect comprising such variables as joy, happiness, relaxation, excitement, and interest; negative affect comprising such variables as stress, anxiety, hostility, fear, and shame; and satisfaction with life comprising variables that represent cognitive evaluations about the self, world, and future. However, the independence of the variables composing the lower levels of the hierarchy is not relevant to the primary issue, which is that there is now consensus in the emotion literature that at the highest level of the hierarchy the SWB construct varies on a single continuum from low positive affect, high negative affect, and low satisfaction with life, to high positive affect, low negative affect, and high satisfaction with life (Diener et al. 1999).

We suggest that both the weak and the strong approaches imply that measures of anxiety and depression also influence positive functioning through the higher order SWB construct. The strong version of the continuum approach further suggests that the lower down level of SWB are also continuums ranging from positive to negative functioning. The present study is specifically concerned with the measurement of depressive and anxious affective, cognitive and bodily states as continuous with their directly opposite states of positive functioning. As such it does not imply that all aspects of functioning are on a single continuum. For example, it is possible that some people "function optimally" as defined by a set of positive measures, despite having psychiatric disorder (Keyes 2007).

Based on the above rationale the next step in the development of a general purpose inventory that can be used to assess these two states but also provide a positive psychological perspective was to adapt two existing tools: the Short Depression-Happiness Scale (SDHS; 
Joseph et al. 2004) and the Inventory Short Form of the Spielberger Anxiety Scale (STAI-6; Marteau \& Bekker 1992). Specifically, the SDHS is a six item self-report scale (containing three negatively worded items, i.e., dissatisfied, cheerless, meaningless, and three positively worded items, i.e., pleased, enjoyable, happy). Someone who endorses the positive items but not the negative items would seem to be functioning in a way which is not only the absence of mental distress but also the presence of positive states. Confirming its validity as a measure of positive functioning, higher scores on the SDHS are associated with higher scores on the WEMWBS $(\mathrm{r}=.73, \mathrm{p}<.001)$ (Tennant et al. 2007).

Although not explicitly developed with a positive psychological use in mind the similar format and mix of positive and negative items of the STAI- 6 to the SDHS suggests that it should equally well serve a positive psychological purpose (Joseph \& Wood 2010). The STAI-6 is a six item self-report scale (containing three negatively worded items, i.e., upset, tense, worried, and three positively items, i.e., content, calm, relaxed).

Both the SDHS and the STAI-6 were developed for use in clinical contexts and are reliable and valid clinical tools for assessing depressive and anxious states, respectively. However, each is limited insofar as its use a general purpose tool, assessing as each does only a portion of the circumplex of affective states (Joseph \& Wood 2010). What is unusual about these two scales, compared to most of the other measures of depression and anxiety available, is that they both contain an equal balance of positive and negatively worded items. Other measures could have been selected but these were favored for their individual brevity and the fact that used in combination the total number of items to be completed was only 12 , which is sufficiently short to be useful to clinicians, such that the measure could be quickly administered, used alongside other traditional measures if required, and for repeated session by session use.

Two modifications were made. First, items were adapted with rewording of the STAI-6 items from present to past tense for consistency with the SDHS, Second, items were preceded by a sentence asking participants to complete the questionnaire with reference to how they had felt during the last week, on a four point scale, 0 (never), 1 (rarely), 2 (sometimes), 3 (often) consistent with the format of the SDHS (see Additional file 1). The frequency of items was asked in recognition that over a period of time people may experience both positive and negative states.

We had five research aims. First, we were concerned to establish the relationship between the two clusters of items adapted from the existing measures, the SDHS and the STAI-6. While problems of depression and anxiety are known to be experientially different, these two states have been found to be strongly associated and difficult to separate empirically, with correlations between measures of depression and anxiety typically in range of $r=0.80$ and above (Feldman 1993; Watson et al. 1995). Approximately 85\% of patients with depression also experience significant symptoms of anxiety. Similarly, comorbid depression occurs in up to $90 \%$ of patients with anxiety disorders (Gorman 1998). There is much common content in measures of depression and anxiety (Endler et al. 1992). Selection of items specific to the constructs of either depression or anxiety can yield empirically separate tools (Stulz \& Crits-Cristoph 2010) but unless specifically developed in this way tend to be highly correlated. As such, at a higher order level these two theoretically separable dimensions may be combined to provide an overall index of positive functioning.

Second, it is important to establish internal-consistency and test-retest reliability in order to show that the items do cohere and responses remain relatively stable over time, 
which would be congruent with the view that the aggregate assessment of well-being across any sample should remain comparatively equal across shorter periods of time, unless those studied experience a particular life event thought to effect well-being.

Third, we formed a nomological net of theoretically related constructs for which correlations with the PFI-12 would demonstrate concurrent validity. Within positive psychology there are two traditions of well-being research, the hedonic and the eudaimonic. These two philosophical traditions underlie research into subjective well-being (SWB) and psychological well-being (PWB), respectively (Joseph \& Wood 2010).

While the distinction between SWB and PWB is of theoretical interest and has helped to define the scope of positive psychology it is not of such interest to practitioner psychologists and therapists, for whom the concepts of depression and anxiety are more relevant to the everyday concerns of their patients. The concepts of anxiety and depression both include elements of SWB and PWB. Sates of depression and anxiety involve a phenomenological mix of SWB and PWB-related experiences. As such the PFI-12 includes items related both to SWB and its constituent parts of positive affect (happy content, calm, relaxed), negative affect (cheerless, upset, tense, worried), life satisfaction (enjoyable, dissatisfied) and to a lesser extent PWB with self-acceptance (pleased) and meaning (meaningless). Thus, despite the different theoretical conceptualizations we would expect strong associations to be found with measures of well-being, particularly with the positive and negative affect components of SWB, but also to a lesser extent with PWB and related variables such as self-efficacy, self-esteem and optimism. We would also expect people high on positive functioning to score highly on a range of other measures related to health such as how they cope with difficulties in their life. People who are more optimally functioning typically use a higher degree of reappraisal coping and forms of coping that actively approach problematic situations, and are more resilient.

It is important to establish concurrent validity with a wide nomological net in order to demonstrate the adaptive functioning of individuals. We also wished to expand our nomological net to include measures of personality. Associations with personality are of theoretical interest and important in establishing the construct validity of a new scale. It should be the case that any association is not so strong that the new measure would be seen as an indicator of personality. As such, some correlations were expected with personality, particularly with the Big Five constellation, since previous research using measures of well-being has shown small to moderate positive associations with extraversion, openness, agreeableness, and conscientiousness, and a moderate to strong negative association with neuroticism (Hayes \& Joseph 2003; McCrae \& Costa 1991; Kotov et al. 2010). Previous research suggests that neuroticism and extraversion have the strongest associations (DeNeve \& Cooper 1998). Given these correlations we would also expect higher positive functioning to be associated with scores on Type D personality consisting of a joint tendency towards negative affectivity (NA) and social inhibition (SI). Individuals high on NA and SI have a distressed, or Type D personality, and are at risk for a range of adverse health outcomes (Denollet 2005). A further personality theory is Gray's model which proposes two separate systems of behavioural inhibition (BIS) and behavioural activation (BAS) (Gray 1970; 1987), such that mental well-being is thought to be related to greater activation and lower inhibition. As such it is important that any new measure should be associated with personality variables in a way consistent with previous research, i.e., greater positive functioning should be associated with: (a) lower scores on neuroticism, 
higher sores on extraversion, openness, agreeableness, and conscientiousness; (b) lower scores on NA and SI; and (c) higher scores on BAS and lower scores on BIS.

Fourth, we considered incremental validity for the positive functioning scale over existing conceptualisations of well-being in terms of predicting perceived stress. It is thought that well-being may be useful in predicting perceived stress (Cohen et al. 1983). Perceived Stress is a congruent temporal state that reflects individuals' state appraisals, of whether current environmental demands exceed their ability to deal with such demands, made in specific reference to current personal well-being (Cohen, et al. 1983; Yap \& Tong 2009). Specifically, we are interested in seeing whether PFI-12 shows incremental validity in predicting current stress after controlling for the two main well-being states, hedonic (positive and negative affect) and eudaimonic (psychological) well-being.

Fifth, we wished to test for discriminant validity with social desirability. It is also important to establish that scores on any new measure are not affected by social desirability, given that we are seeking to establish that the PFI-12 does not show signs of distorted responding but provides accurate assessments.

In these five ways we sought to find additional evidence for the validity of the PFI-12 as a measure of mental well-being that will be useful to clinicians, psychotherapists and researchers who wish to look beyond the traditional spotlight of psychopathology to the positive psychological ways in which people can function.

\section{Method}

Data were collected in two different student samples. Participants were volunteers from the University experiment participation scheme where students take part in studies in return for being able to recruit participants to their own research projects. Studies were advertised and volunteers signed up and completed the studies online via an electronic survey system. Ethical approval was obtained from the University Research Ethics Committee prior to data collection.

Respondents provided consent via a first page of the electronic sheet on which they had to indicate agreement before proceeding or otherwise exit the survey. The consent form contained statements and direction regarding the nature of the study, anonymity of the data, withdrawal both within and after the experiment participation, how the data would be stored in a coded form, how to obtain the results of the study if required, and intended use, length of storage and disposal of data. To facilitate withdrawal from the study at a date later than the time of the study participation, respondents were asked to provide a Personal Identification Number (PIN) as part of the survey. This PIN was created by the respondent, recorded on the survey, which then be used at a later date to identify a set of responses to be removed from the survey if required. No respondents asked for their responses to be removed. Records of signing-up to the electronic survey system were used to facilitate the awarding of credit to the participation scheme and used to record consent had been given by each participant. The electronic survey system was also set up so respondents had to answer all questions. Participants were informed that the data for participation in the study could not, and therefore would not, be paired up with the coded data. The use of this PIN was used to identify respondents for a follow-up study examining the test-retest data, with respondents contacted through the electronic survey system using records of their participation in the earlier experiment, for which further credit was awarded. 


\section{Sample 1}

The sample comprised two hundred and forty-two undergraduates (45 males and 197 females) aged 18 to 42 years $(M=20.20$ years, $S D=3.7)$. Respondents came from a variety of cultural backgrounds (Caucasian, 68.2\%; South Asian, 14.9\%; East Asian, 2.1\%; Black, $8.3 \%$; mixed race $4.5 \%$; other, $2.1 \%$ ).

Participants in this sample had all signed up to experimental studies unrelated to the current project but which provided the opportunity for us to ask them to complete the PFI-12. Data on the PFI-12 were subsequently pooled for the purpose of conducting pilot correlational analysis between the two-six item scales.

\section{Sample 2}

The sample comprised three hundred and one undergraduates (75 males and 226 females) aged 18 to 47 years $(M=20.05$ years, $S D=3.4)$. Respondents came from a variety of cultural backgrounds (Caucasian, 66.4\%; South Asian, 14.6\%; East Asian, 3.3\%; Middle Eastern, $0.3 \%$, Black, $7.6 \%$; mixed race, $4.3 \%$; other, $3.3 \%$ ). This study was undertaken in a different year to the sample collected in Sample 1 and data were collected with the purpose of providing convergent validity data.

As well as the PFI-12 respondents completed other well established psychometric measures with which to assess validity.

Five measures were used to assess social and psychological functioning.

First, the 20-item Positive and Negative Affect Scales (PANAS; Watson et al. 1988) was used to assess positive and negative mood states. Second, the 18-item Scales of Psychological Well-Being (Ryff \& Keyes 1995) was used to assess psychological well-being (three items per dimension: autonomy, environmental mastery, positive relations with others, personal growth, purpose in life, and self-acceptance). Factor analysis studies confirm that these six dimensions form a single component that is distinct from reports of hedonic well-being (Linley et al. 2009). As such, only the total scale score was used in the present study. Third, the 8-item New General Self-Efficacy Scale (Chen et al. 2001) was used to assess self-efficacy as a belief in overall competence to attain the required performance across a variety of achievement situations. Fourth, the 10-item Rosenberg Self-Esteem Scale (Rosenberg 1965) was used to assess global self-esteem. Fifth, the 10-item Life Orientation Test - Revised (LOT-R; Scheier et al. 1994) was used to assess an individual's level of dispositional optimism. Six of the items are used to measure optimism (e.g. "I'm always optimistic about my future"), with four of the items used as filler items.

Five measures were used to assess health, stress, coping and resilience.

First, the 14-item Physical Health Questionnaire (PHQ) (Schat et al. 2005) was used to assess four dimensions of somatic symptoms: gastrointestinal problems ( 4 items; e.g. How often have you suffered from an upset stomach (indigestion)?), headaches (3 items; How often have you experienced headaches?), sleep disturbances (4 items; "How often have you had difficulty getting to sleep at night?"), and respiratory illness (3 items; e.g. When you have a bad cold or flu, how often does it last longer than it should?). Second, the 10-item Perceived Stress Scale (Cohen et al. 1983) was used to assess perception of stress (e.g. In the last month, how often have you felt nervous and "stressed"?). Third, coping was measured via the 16-item Functional Dimensions of Coping Scale (Ferguson \& Cox 1997) which assesses four dimensions of coping; approach, avoidance, reappraisal, and emotional regulation. Fourth, the 25-item Psychological Resilience Scale (Wagnild \& Young 1993) was 
used to assess resilience via the capacity to withstand stress and create meaning from challenges. Psychometrically there is evidence of a two factor structure to the scales, comprising Personal Competence (self-reliance, independence, determination, invincibility, mastery, resourcefulness and perseverance) and Acceptance of Self and Life (adaptability, balance, flexibility and a balanced perspective on life) (Portzky et al. 2010; Wagnild \& Young 1993). For the purpose of this study we computed the two subscales of Personal Competence and Acceptance of Self and Life. Fifth, the 6-item Brief Resilience Scale (Smith et al. 2008) was used to measure an individual's ability to 'bounce back' to stressful situations or adversity.

Three measures were used to assess personality.

First, the 60-item Short Five (Konstabel et al. 2011) was used to measure the five factor model of personality, from which scores of Neuroticism, Extraversion, Openness to Experience, Conscientiousness and Agreeableness can be derived. Second, the 14-item Type D Personality Scale (DS14) (Denollet 2005) was used to assess negative affectivity and social inhibition. Third, the 24-item BIS/BAS Scales (Carver \& White 1994) were used to measure Gray's model of personality. The BIS scale contains seven items assessing anxiety sensitivity to events. The BAS scale includes 13 items that are subdivided into Drive, Fun Seeking (both four items) and Reward Responsiveness (five items). Four items are used as filler items.

Finally, the 6-item Lie subscale of the Abbreviated form of the Revised Eysenck Personality Questionnaire - Short-Form (Francis et al. 1992) was used to assess social desirability. With these additional data we specifically wished to test the PFI-12 association with social desirability.

In addition 168 of these respondents in Sample 2 (36 males, 132 females) aged between 18 and 47 years $(M=19.96 ; S D=3.0$, at time 1$)$ again completed the PFI-12 at six months to test for its stability over time.

\section{Results}

\section{Association between depression and anxiety}

We were concerned to ascertain the relationship of the two six-item scales. Both six-item clusters were correlated at $r=.79, p<.001$ for sample $1, r=.76, p<.001$ for sample 2 and $r=.79, p<.001$ for sample 2 at time 2 . These results are consistent with previous research which has shown that broad based measures of depression and anxiety generally do not tap distinct construct and supports the summation of both scales to provide an overall index, the 12-item Positive Functioning Inventory (PFI-12) with higher scores indicating greater positive functioning. The highest possible score on the PFI-12 is 36 and indicates full endorsement of the six positive items (pleased, enjoyable, happy content, calm, relaxed) and a lack of endorsement of the six negative items (dissatisfied, cheerless, meaningless, upset, tense, worried). Conversely, the lowest possible score of 0 would indicate a lack of endorsement of the positive items and full endorsement of the negative items. As such practitioners are able to plot the trajectory of client's recovery across the full spectrum of functioning, with higher scores indicating greater positive functioning.

Internal consistency and test-retest reliability

Internal consistency reliability of the PFI-12 was found to be acceptable in all three samples $(\alpha=.87$ for sample $1, \alpha=.86$ for sample 2 , and $\alpha=.88$ for sample 2 at time 2 ). 
These results indicate that the measure has a high degree of internal coherence. For the sub group of sample 2 who completed the measure at two occasions, separated by six months, scores on the PFI-12 at time $1(M=23.96 ; S D=7.0)$ were associated with scores at time $2(M=21.41, S D=7.9)$ with the interclass correlation coefficient $=.62, p<.001)$, being above the 0.60 minimum criteria suggested by Chinn (1991). These results indicate that the measure is moderately stable over time as would be expected in a state measure of well-being but not so stable that it would be considered a trait.

\section{Construct validity}

With sample 2, higher scores on the PFI-12 were associated with lower scores on PANAS negative affect $(r=-.63, p<.001)$ and higher scores on PANAS positive affect $(r=.50$, $p<.001)$. Higher scores on the PFI-12 were associated with higher scores on the total PWB $(r=.47, p<.001)$. Higher scores were also associated with higher self-efficacy $(r=.37, p<.001)$ self-esteem $(r=.48, p<.001)$, and optimism $(r=.45, p<.001)$.

Higher scores on the PFI-12 were associated with better health $(r=-.25, \mathrm{p}<.01)$, lower scores on perceived stress $(r=-.55, p<.001)$, higher scores on approach coping $(r=.21$, $p<.01)$, emotional regulation coping $(r=.35, p<.01)$ and reappraisal coping $(r=.29$, $p<.01)$. No association was found for avoidance coping $(r=.10$, ns). Higher scores on the PFI-12 were associated with personal competence resilience $(r=.22, p<.01)$, acceptance of life and self resilience $(r=.24, p<.01)$, and resilience ability to bounce back $(r=.33, p<.01)$.

As predicted higher scores on the PFI-12 were moderately associated with lower scores on neuroticism $(r=-.49, p<.001)$ and higher scores on extraversion $(r=.37, p<.001)$. Weak associations were also found for openness $(r=.14, p<.05)$ and conscientiousness $(r=.12, p<.05)$ although no association was found for agreeableness $(r=.05$, ns). Consistent with our findings for neuroticism and extraversion, higher scores on the PFI-12 were associated with lower scores on negative affectivity $(r=-.48, \mathrm{p}<.001)$ and social inhibition $(r=-.29, p<.001)$ on the Type D Personality Scale. An association was also found between higher scores on the PFI-12 and higher scores on the BAS drive $(r=.12, \quad p$ $<.05)$, BAS fun seeking $(r=.22, p<.05)$, and lower scores on the BIS $(r=-.36, p<.001)$ subscales. No association was found for the BAS reward responsiveness $(\mathrm{r}=.10, \mathrm{~ns})$.

\section{Incremental Validity}

We were interested in seeing whether PFI-12 shows incremental validity in predicting current stress after controlling for the two main extant well-being states, hedonic (positive and negative affect) and eudaimonic (psychological) well-being. In the first step, the inclusion of measures of positive affect, negative affect and psychological well-being in predicting perceived stress reached statistical significance $(R=.65, R 2=.43$, Adjusted $R 2=.42$, $F 3,173=42.86, p<.001)$, with positive affect $(\beta=-.22, p=.001)$ and negative affect $(\beta=.50$, $p<.001$ demonstrating statistical significant regression coefficients. In the second step, the inclusion of the PFI-12 was found to provide a significant R2 change ( $\mathrm{R} 2$ change $=.015$, $\mathrm{F}$ change1, $172=4.56, \mathrm{p}=.034)$ with positive functioning demonstrating a statistical significant regression coefficient $(\beta=-.18, p=.034)$.

\section{Discriminant validity}

For sub group of sample 2, no association was found between scores on the PFI-12 and lie scale scores $(r=.02$, ns). 


\section{Discussion}

Reliability and validity estimates supported the psychometrics of the PFI-12 with college women and men, as evidenced by tests for its internal consistency reliability, test-retest reliability, and construct and discriminant validity with a number of other measures chosen to assess the characteristics of people who are functioning positively. As expected, effect sizes were highest with well-being, negative and positive affect, stress, self-efficacy and self-esteem. With secondary variables of coping and resilience, medium effect sizes were found providing evidence for convergent validity. As such the PFI-12 appears to provide an index of general psychological health, with lower scores indicating the presence of dysfunction, and higher scores the presence of positive functioning.

Associations were also found with personality variables as expected although none of the associations tested for were found to be so high that the new measure could be considered synonymous with personality. The highest personality effect sizes were with neuroticism, accounting for just under $25 \%$ of the variance and with extraversion, accounting for $14 \%$ of the variance. Further psychometric work could expand the nomological net wider to test for convergent validity with other theoretically related constructs derived from the positive psychology field that are known to be associated with well-being, such as authenticity (Wood et al. 2008) and gratitude (Wood et al. 2009).

We suggest that the PFI-12 promises to have utility for practitioners whose concern is not only with the treatment of distress and dysfunction but also the facilitation of positive functioning, in three ways. First, such a tool is likely to be of particular interest to therapists working from a humanistic perspective and whose approach already encompasses the idea that therapy is a way to facilitate well-being (Joseph \& Linley 2006). Such therapists may find the scale useful as it provides a measure more consistent with their theoretical orientation. Second, it is becoming increasingly recognised that there is a need for scientific studies into the effectiveness of therapy. Such studies will benefit from the use of measures of wellbeing. Newer therapies in the positive psychological tradition will also find the tool useful (Joseph \& Linley 2006). Third, the PFI-12 can also be used as a research tool with which to assess the determinants of well-being and the factors that promote positive outcomes in therapy. For example, although, it is known that the therapeutic alliance is one of the important ingredients in therapy, we only know this in relation to recovery from symptoms. It remains to be seen to what extent the relationship is also important in promoting positive change.

There are several advantages to the PFI-12. First, even though it is an adaption of two existing measures, it remains relatively brief. As such, it is likely to maximize response rates, and minimize the number of response errors and unanswered items. Its brevity also means that the PFI-12 can be easily accommodated within a wider battery of measures when other issues are also a target of assessment. Second, the PFI-12 provides a novel conceptualisation of well-being as a spectrum of functioning. As such it is a useful tool for practitioners and researchers who wish to assess the full range of functioning and not be restricted by either floor or ceiling effects. By and large, new measures in the positive psychology literature tend be statistically unipolar. As such, despite the popularity of such tools among positive psychologists and others interested in human flourishing, their use by practitioners and researchers within clinical psychology is limited because of floor effects. Third, although based on existing measures which relate to current conceptions of dysfunction the aim in developing the PFI-12 is to reconceptualise these clinical phenomena 
in a new way that provides a non-medicalized view of functioning. The PFI-12 accommodates existing clinical language of depression and anxiety within a statistically bipolar positive psychological conceptualisation. This is advantageous because the relevance of the tool to clinicians is clear.

Scores on the PFI-12 can provide a useful summary of a client's progress. As with all such tools, clinical judgment is always important. Items are asked in relation to their frequency. One might expect that most people who are functioning well in their lives will score relatively highly on the tool indicating that for most of the week they experienced more positive states than negative states. However, we would suggest that clinicians must be cautious in interpreting the scores of those who consistently score at the maximum. We cannot rule out the possibility that such a scoring pattern may in fact reflect an illusory or self-deceptive state in some. It is also important in the interpretation of scores to take into account the context of a person's life and what affective, cognitive and bodily states are likely to be adaptive given their unique circumstances.

As already mentioned, the concepts of depression and anxiety cut across the positive psychology constructs of (hedonic) subjective and (eudaimonic) psychological wellbeing. While positive psychologists may consider SWB and PWB as separate topics for research, therapeutically it has long been recognised in the description of depressive and anxious states that SWB and PWB are related and difficult to distinguish.

There are limitations to the study and a need for further research. First, the sample was comprised of undergraduate students and not a clinical sample. As such there is further evidence required to validate its use as a clinical tool. However, we would note that the scale is adapted from two previous established clinical measures of anxiety and depression and as such there is no reason to think that it would not perform as expected. Second, no evidence of change was provided due to intervention. Further evidence is also needed to show that the scale will be a sensitive measure of change over sessions in the facilitation of positive functioning. Finally, as yet there has been little research attention on how positive psychology applications apply to the most deeply distressed and dysfunctional. For example, do traditional treatments for the alleviation of psychological distress also facilitate positive functioning? Are the exercises and techniques developed by positive psychologists for the promotion of well-being also helpful for the alleviation of psychological distress? The PFI-12 is one research tool that can facilitate this new avenue of research.

In conclusion the PFI-12 performed well against accepted criteria, is reliable and valid, and easy to administer. It is especially useful to practitioners because it addresses the traditional need to assess states of distress and dysfunction but also accommodates the perspective of positive psychology, allowing clinicians to conceptualise therapy as consisting of both the alleviation of symptoms and the promotion of well-being.

\section{Endnotes}

${ }^{a}$ Note that all 301 respondents completed the PF-12 but the full battery of measures were not administered to all respondents. Only 177 (41 males, 136 females) aged between 18 and 34 years $(\mathrm{M}=19.86$; $\mathrm{SD}=2.23)$ also completed the The Perceived Stress Scale, Physical Health Questionnaire (PHQ), The Type D Personality Scale, Life Orientation Test - Revised, The Psychological Resilience Scale, The Brief Resilience Scale and the Lie subscale of the Abbreviated form of the Revised Eysenck Personality Questionnaire - Short-form. 


\section{Additional file}

Additional file 1: Positive Functioning Inventory-12 (PFI-12).

\section{Acknowledgements}

The authors wish to thank Professor Alex Wood for his advice.

\section{Author details}

${ }^{1}$ School of Education, University of Nottingham, NG8 1BB, UK. ${ }^{2}$ School of Psychology, Leicester University, Leicester LE1 9HN, UK

Received: 20 February 2014 Accepted: 16 July 2014

Published online: 05 September 2014

\section{References}

Beck, AT, Ward, CH, Mendelson, M, Mock, JE, \& Erbaugh, JK. (1961). An inventory for measuring depression. Archives of General Psychiatry, 4, 561-571.

Beck, AT, Epstein, N, Brown, G, \& Steer, RA. (1988). An inventory for measuring clinical anxiety: psychometric properties. Journal of Consulting and Clinical Psychology, 56(6), 893

Carver, CS, \& White, TL. (1994). Behavioral inhibition, behavioral activation, and affective responses to impending reward and punishment: The BIS/BAS scales. Journal of Personality and Social Psychology, 67, 319-333.

Chen, G, Gully, SM, \& Eden, D. (2001). Validation of a new General Self-Efficacy Scale. Organizational Research Methods, 4, 62-83.

Chida, Y, \& Steptoe, A. (2008). Positive psychological well-being and mortality: a quantitative review of prospective observational studies. Psychosomatic Medicine, 70, 741-756.

Chinn, S. (1991). Repeatability and method comparison. Thorax, 46, 454-456.

Cohen, S, Kamarck, T, \& Mermelstein, R. (1983). A Global Measure of Perceived Stress. Journal of Health and Social Behavior, $24,385-396$

DeNeve, KM, \& Cooper, H. (1998). The happy personality: a meta-analysis of 137 personality traits and subjective well-being. Psychological Bulletin, 124, 197-229.

Denollet, J. (2005). DS14: standard assessment of negative affectivity, social inhibition, and Type D personality. Psychosomatic Medicine, 67, 89-97.

Diener, E, Emmons, RA, Larsen, RJ, \& Griffin, S. (1985). The Satisfaction with Life Scale. Journal of Personality Assessment, $49,71-75$.

Diener, E, Suh, EM, Lucas, RE, \& Smith, H. (1999). Subjective well-being: Three decades of progress. Psychological Bulletin, $125,276-302$.

Ekkekakis, P. (2013). The measurement of affect, mood, and emotion: A guide for health-behavioral research. Cambridge: Cambridge University Press.

Endler, N, Cox, B, Parker, J, \& Bagby, M. (1992). Self-reports of depression and state-trait anxiety: Evidence for differential assessment. Journal of Personality and Social Psychology, 63, 832-838.

Feldman, LA. (1993). Distinguishing depression and anxiety in self-report: Evidence from confirmatory factor analysis on nonclinical and clinical samples. Journal of Consulting and Clinical Psychology, 61, 631-638.

Ferguson, E, \& Cox, T. (1997). The functional dimensions of coping scale: theory, reliability and validity. British Journal of Health Psychology, 2, 109-129.

Fischer, J, \& Corcoran, K (Eds.). (2007). Measures for clinical practice and research: a sourcebook. New York: Oxford University Press.

Francis, LJ, Brown, LB, \& Philipchalk, R. (1992). The development of an abbreviated form of the Revised Eysenck Personality Questionnaire (EPQR-A): Its use among students in England, Canada, the USA and Australia. Pers Individ Differences, 13(4), 443-449.

Gorman, JM. (1998). Comorbid depression and anxiety spectrum disorders. Depression and Anxiety, 4, 160-168.

Gray, JA. (1970). The psychophysiological basis of introversion \pm extraversion. Behaviour Research and Therapy, 8, 249-266.

Gray, JA. (1987). The psychology of fear and stress (2nd ed.). Cambridge: Cambridge University Press.

Hayes, N, \& Joseph, S. (2003). Big 5 correlates of three measures of subjective well being. Personality and Individual Differences, 34, 723-727.

Howell, RT, Kern, ML, \& Lyubomirsky, S. (2007). Health benefits: meta-analytically determining the impact of well-being on objective health outcomes. Health Psychology Review, 1, 83-136.

Joseph, S, \& Lewis, CA. (1998). The Depression - Happiness Scale: Reliability and validity of a bipolar self-report scale. Journal of Clinical Psychology, 54, 537-544.

Joseph, S, \& Linley, PA. (2006). Positive therapy: a meta-theoretical approach to positive psychological practice. London: Routledge.

Joseph, S, \& Wood, A. (2010). Assessment of positive functioning in clinical psychology: Theoretical and practical issues. Clinical Psychology Review, 30, 830-838.

Joseph, S, Linley, PA, Harwood, J, Lewis, CA, \& McCollam, P. (2004). Rapid assessment of well-being: The short depressionhappiness scale (SDHS). Psychology and Psychotherapy: Theory, Research and Practice, 77, 463-478.

Keyes, CLM. (2007). Promoting and Protecting Mental Health as Flourishing: A Complementary Strategy for Improving National Mental Health. American Psychologist, 62, 95-108.

Konstabel, K, Lonnqvist, JE, Walkowitz, G, Konstabel, K, \& Verkasalo, M. (2011). The 'Short Five' (S5). Measuring personality traits using comprehensive single items. European Journal of Personality, 26, 13-29.

Kotov, R, Gamez, W, Schmidt, F, \& Watson, D. (2010). Linking "big" personality traits to anxiety, depressive, and substance use disorders: a meta-analysis. Psychological Bulletin, 136, 768-821.

Layard, R. (2006). The depression report: A new deal for depression and anxiety disorders (No. 15). LSE: Centre for Economic Performance. 
Linley, PA, Maltby, J, Wood, AM, Osborne, G, \& Hurling, R. (2009). Measuring happiness: The higher order factor structure of subjective and psychological well-being measures. Personality and Individual Differences, 47, 878-884.

Lovibond, PF, \& Lovibond, SH. (1995). The structure of negative emotional states: Comparison of the Depression Anxiety and Stress Scales (DASS) with the Beck Depression and Anxiety Inventories. Behaviour Research and Therapy, 33, 335-342.

Lyubomirsky, S, \& Lepper, H. (1999). A measure of subjective happiness: preliminary reliability and construct validation. Social Indicators Research, 46, 137-155.

Maddux, JE, Snyder, CR, \& Lopez, SJ. (2004). Toward a positive clinical psychology: Deconstructing the illness ideology and constructing an ideology of human strengths and potential. In PA Linley \& S Joseph (Eds.), Positive psychology in practice (pp. 320-334). Hoboeken: Wiley.

Marteau, TM, \& Bekker, H. (1992). The development of a six-item short-form of the state scale of the Spielberger State-Trait Anxiety Inventory (STAI). Br J Clin Psychol, 31, 301-306.

McCrae, RR, \& Costa, PT, Jr. (1991). Adding Liebe und Arbeit: The Full Five-Factor Model and Well-Being. Personality and Social Psychology Bulletin, 17, 227-232.

Parkinson, GR. (2002). User's guide for Duke Health Measures. Durham, NC: Department of Community and Family Medicine, Duke University Medical Center.

Pavot, W, \& Diener, E. (2004). Findings on subjective well-being: Applications to public policy, clinical interventions, and education. In PA Linley \& S Joseph (Eds.), Positive Psychology in Practice (pp. 679-692). Hoboeken: Wiley.

Portzky, M, Wagnild, G, De Bacquer, D, \& Audenaert, K. (2010). Psychometric evaluation of the Dutch Resilience Scale RS-nl on 3265 healthy participants: a confirmation of the association between age and resilience found with the Swedish version. Scandinavian Journal of Caring Sciences, 24, 86-92.

Rashid, T, \& Ostermann, RF. (2009). Strength-based assessment in clinical practice. Journal of Clinical Psychology, 65, 488-498. Rosenberg, M. (1965). Society and the adolescent self-image. Princeton, NJ: Princeton University Press.

Russell, JA. (1980). A circumplex model of affect. Journal of Personality and Social Psychology, 39, 1161-1178.

Russell, J, \& Barrett, L. (1998). Independence and bipolarity in the structure of current affect. Journal of Personality and Social Psychology, 74, 967-984.

Ryff, CD, \& Keyes, CL. (1995). The structure of psychological well-being revisited. Journal of Personality and Social Psychology, 69, 719-727.

Schat, A, Kelloway, EK, \& Desmarais, S. (2005). The Physical Health Questionnaire (PHQ): Construct Validation of a Self-Report Scale of Somatic Symptoms. Journal of Occupational Health Psychology, 10, 363-381.

Scheier, MF, Carver, CS, \& Bridges, MW. (1994). Distinguishing optimism from neuroticism (and trait anxiety, self-mastery, and self-esteem): A re-evaluation of the Life Orientation Test. Journal of Personality and Social Psychology, 67, 1063-1078.

Seligman, MEP, \& Csikszentmihalyi, M. (2000). Positive psychology: An introduction. American Psychologist, 55, 5-14.

Seligman, MEP, Steen, TA, Park, N, \& Peterson, C. (2005). Positive psychology progress: Empirical validation of interventions. American Psychologist, 60, 410-421.

Seligman, MEP, Rashid, T, \& Parks, AC. (2008). Positive Psychotherapy. Am Psychol, 61, 774-788,

Smith, BW, Dalen, J, Wiggins, K, Tooley, E, Christopher, P, \& Bernard, J. (2008). The Brief Resilience Scale: Assessing the ability to bounce back. International Journal of Behavioral Medicine, 15, 194-200.

Stulz, N, \& Crits-Cristoph, P. (2010). Distinguishing anxiety and depression in self-report: purification of the beck anxiety inventory and beck depression inventory-ll. Journal of Clinical Psychology, 66, 927-940.

Tennant, R, Hiller, L, Fishwick, R, Platt, S, Joseph, S, Weich, S, \& Stewart-Brown, S. (2007). The Warwick-Edinburgh Mental Well Being Scale (WEMWBS): Development and UK validation. Health and Quality of Life Outcomes, 5, 63.

Wagnild, GM, \& Young, HM. (1993). Development and psychometric evaluation of the resilience scale. Journal of Nursing Measurement, 1, 165-178.

Watson, D, Clark, LA, \& Tellegen, A. (1988). Development and validation of brief measures of positive and negative affect: The PANAS scales. Journal of Personality and Social Psychology, 54, 1063-1070.

Watson, D, Weber, K, Assenheimer, J, Clark, L, Strauss, M, \& McCormick, R. (1995). Testing a Tripartite model: I: Evaluating the convergent and discriminant validity of anxiety and depression symptom scales. Journal of Abnormal Psychology, 104, 3-14.

Wood, AM, \& Joseph, S. (2010). The absence of positive psychological (eudemonic) well-being as a risk factor for depression: A ten year cohort study. Journal of Affective Disorders, 122, 213-217.

Wood, AM, Linley, PA, Maltby, J, Baliousis, M, \& Joseph, S. (2008). The authentic personality: A theoretical and empirical conceptualization and the development of the authenticity scale. Journal of Counseling Psychology, 55, 385-399.

Wood, AM, Joseph, S, \& Maltby, J. (2009). Gratitude Predicts Psychological Well-Being above the Big Five Facets. Personality and Individual Differences, 46, 443-447.

Yap, AJ, \& Tong, EMW. (2009). The Appraisal Rebound Effect: Cognitive Appraisals on the Rebound. Personality and Social Psychology Bulletin, 35, 195-221.

Zigmond, AS, \& Snaith, RP. (1983). The Hospital anxiety and Depression Scale. Acta Psychiatricas Scandinavica, 67, 361-370.

doi:10.1186/s13612-014-0015-6

Cite this article as: Joseph and Maltby: Positive functioning inventory: initial validation of a 12-item self-report measure

of well-being. Psychology of Well-Being: Theory, Research and Practice 2014 4:15. 